\title{
Prevalence of Psychoactive Substance Use Among Nigerian Male Commercial Vehicle Drivers Selected from The Three Major Ethnic Groups in Nigeria
}

\author{
Tochukwu Frank Egwuatu*, Onyekachi Ogbonnaya Iroanya, Khalid Olajide Adekoya \\ Department of Cell Biology and Genetics, Faculty of Science, University of Lagos, Nigeria \\ Corresponding author* \\ tochukwufrankegwuatu@gmail.com
}

Manuscript received: 16 June, 2020. Revision accepted: 23 July, 2020. Published: 26 July, 2020.

\begin{abstract}
The use of psychoactive substances for several reasons by commercial vehicle drivers has been documented as a major cause of increased incidences of traffic accidents globally. The study aimed at determining the prevalence of psychoactive substance use among Nigerian male commercial vehicle drivers randomly selected from the three major ethnic groups in Nigeria (Igbo, Hausa and Yoruba). A structured questionnaire which included data on sociodemographic status and psychoactive drug use history was introduced to all participants. Generic Multi-Drug Urine Dip Card Test Kit and Shimadzu chromatograph system (Shimadzu Corporation, Kyoto, Japan) were used for toxicological analyses. Out of the 264 Nigerian male commercial vehicle drivers who participated in this study, 111(42.0\%) had used illicit drugs in recent times, 55(20.8\%) took drugs for improved energy and 90(34.1\%) felt high after using illicit drugs. Marijuana was the most abused substance; 77(29.2\%) followed by benzodiazepine; 34(12.9) across all ethnicities studied. Across all ethnicities, HPLC revealed that the Hausa drivers had the highest concentration of psychoactive substances in their urine samples. These findings, reiterates the need for the Nigerian anti-drug agency to develop new strategies in conducting a repressive drug war and extending the same to bus stations.
\end{abstract}

Keywords: Psychoactive drug; Accident; Drivers; Forensic toxicology; Nigeria.

\section{INTRODUCTION}

Forensic toxicology is the science which applies the principles of analytical chemistry and clinical toxicology for medico-legal purpose where the results are likely to be used in court (Wyman, 2012; Smith and Bluth, 2016). Forensic toxicology covers human performances such as impaired driving due to drug consumption, use of steroids by athletes and workplace drug testing (Jenkins, 2010, Wyman, 2012). Substance abuse is a patterned use of a drug in which the user consumes the substance in amounts or with methods which are harmful to themselves or others (Nutt et al., 2010). Psychoactive substances are classified into three main groups: those that alter perception, those that stimulate the brain and those that depress it (Vij, 2011). In 2018, the prevalence of any drug use in Nigeria is estimated at 14.4 per cent or 14.3 million people aged between 15 and 64 years and the extent of drug use in Nigeria is comparatively high when compared with the 2016 global annual prevalence of any drug use of 5.6 per cent among the adult population (UNODC, 2018). Psychoactive substances are prevalent in biological samples taken from injured drivers at the emergency units of several hospitals. Several studies have reported high prevalence rates not only of alcohol but also of medicinal and illicit drugs in such samples (Bogstrand et al., 2011; Drummer et al., 2012). A lot is known about the magnitude of problems associated with drink-driving and the effectiveness of related countermeasures, but much less is known about driving under the influence of other psychoactive substances. Currently, there are no global estimates of deaths resulting from drug-driving, nor is the prevalence of drug-driving known. However, growing recognition of the problem of drug-driving has led to increased focus on this area among road safety policy-makers and researchers (EMCDDA, 2015). The issues linked with driving under the influence of alcohol and narcotic substances are subject to continuous interest not only for legal practitioners, but also for the police, prosecution, and medico-legal teams (EMCDDA, 2012). The use of psychoactive substances among commercial vehicle drivers is not only increasing but strangely predisposing the drivers and their passengers to health risks. There is however paucity of empirical data on the factors associated with this increase (Yunusa et al., 2017). Road traffic injuries claim more than 1.2 million lives each year and have a huge impact on health and development (Gopalakrishnan, 2012). Road traffic accidents are the leading cause of death among young 
people aged between 15 and 29 years. The risk of a road traffic death varies significantly by region, and there has been little change in the regional rates of death since 2010. Injury and deaths arising from road traffic accidents are a major public health problem in developing countries where more than $85 \%$ of all deaths and $90 \%$ of disability-adjusted life years were lost from road traffic injuries (Nantulya and Reich, 2002). Road traffic accidents have emerged as an important public health issue which needs to be tackled by a multidisciplinary approach (Gopalakrishnan, 2012). The highest rates are still in the African Region, while the European Region has a rate far below the global average (9.3 per 100000 populations, relative to the global rate of 17.4 (WHO, 2015). Almost half of all deaths on the world's roads are among those with the least protectionmotorcyclists, cyclists and pedestrians (WHO, 2015). Motor vehicle accident is a significant cause of mortality and morbidity in Nigeria, and driving under the influence of psychoactive substances to improve their performance and keep sleep at bay for as long as possible. The causes of motor vehicle accidents are varied and multi-factorial. However, the use of psychoactive substances plays a major role in the occurrence of motor vehicle accidents. Studies in Nigeria and other countries have shown a high prevalence of use of psychoactive substances, among various categories of drivers (Adekoya et al., 2011). Pela (1989) documented the use following drugs in decreasing order of frequency: alcohol, cigarettes, stimulants, cannabis (Indian hemp) and sedativehypnotics by adolescents in Benin, Nigeria. Males had been documented to have a higher prevalence of use of all the psychoactive substances (Igwe et al., 2009; Oshodi et al., 2010). The lifetime use of caffeine, alcohol, and tobacco has been noted to be significantly associated with male gender (Akanni and Adayonfo, 2015). A significant substance abuse problem in the undergraduate population in Botswana with alcohol being the most commonly used substance has also been reported (Olashore et al., 2018). The prevalence of psychoactive substances use among middle and high school students in the North Center of Morocco was comparable to the national prevalence. Hence, there is need to initiate psychoactive substance prevention programs among school students which should be based on factors associated with psychoactive substance use (Zarrouq et al., 2016). The study aimed at determining the prevalence of psychoactive substance use among Nigerian male commercial vehicle drivers randomly selected from the three major ethnic groups in Nigeria (Igbo, Hausa and Yoruba).

\section{MATERIAL AND METHOD}

\section{Study design, location and population}

This was a cross sectional study which employed both experimental and quantitative research design. The study adopted the purpose/judgmental sampling technique. The technique is a non-probability sampling method because the participants of interest which were drivers were selected from different motor parks located in Anambra, Abia (Igbo-speaking), Kaduna, Sokoto (Hausa-speaking), Oyo and Lagos States (Yorubaspeaking) ethnic group in Nigeria. The target population for the study was the male commercial driver's population (motorcycle riders, tricycle riders, taxi and bus drivers).

\section{Ethical approval}

Prior to sample collection, the research details were explained to all the participants after which they willingly consented to participate in the study. Questionnaires were completed by all the study participants and informed written consent was obtained. The study protocol was approved by the Health Research Ethics Committee (CMULHREC number: CMUL/HREC/05/18/349) of the University of Lagos, Nigeria. Permission was also requested and obtained from the National Union of Road Transport Workers (NURTW) in all sample States before the study was conducted.

\section{Sample and Data Collection}

Approximately $15 \mathrm{ml}$ of urine was collected from each driver who participated in this study using sterile sample bottles. An on-site urinalysis was carried out using $5 \mathrm{ml}$ of each urine sample to determine the general health status of the subjects. Afterwards, the remaining urine samples of the subjects were transported to the laboratory for toxicological analysis. Each driver in the various motor parks was interviewed until the desired sample size was attained. They responded to a sociodemographics and a semi-structured pro forma, which sought information on the type of substance commonly used, reasons for their use, usage frequency and related accident history.

\section{Toxicological Analysis}

The urine samples were tested using 10 Panel Generic Multi Drug Urine Dip Card Test Kit. The kit tested for the presence of ten psychoactive drugs (methamphetamine (METH), cocaine (COC), oxycodone (OXY), morphine (MOP), amphetamine (AMP), methadone (MTD) and barbiturates (BAR), marijuana (THC), Benzodiazepines (BZO) and phencyclidine (PCP)). The tip of the kit was dipped into the urine sample and allowed to wait for minimum of 10 seconds. The kit had a positive and negative panel for each drug. Each sample that tested positive to a particular drug would indicate a red line while negative showed two lines. 


\section{Quantification of Drugs in Polysubstance Users}

High performance liquid Chromatographic (HPLC) analysis was performed with the use of a Shimadzu liquid chromatograph equipped with a model LC2010AHT gradient pump, CBM-20A system controller and Shimadzu CLASS-VP UV-Visible detector (Shimadzu Corporation, Kyoto, Japan). All chemicals and reference standards (morphine, phencyclidine, benzodiazepine and $\Delta 9$-Tetrahydrocannabinol solution used for the HPLC analysis were all of analytical grade. purchased from Sigma-Aldrich. The chromatographic separations were performed using a Sepax BR-C18 column (250×4.6 mm; Sepax Technologies, Inc.) with 5$\mu \mathrm{m}$ particle diameter. The column temperature was set at $25^{\circ} \mathrm{C}$, and the mobile phase was water $(0.1 \%$ formic acid) and Acetonitrile (0.1\% formic acid). This was delivered at a flow rate of $0.8 \mathrm{ml} / \mathrm{min}$. The wavelength of the detector was set at $254 \mathrm{~nm}$, and the injection volume of each extract was either $10 \mu \mathrm{l}$ for assay determination.

\section{Data Analysis}

All numerical data collected were statistically analysed using Statistically Package for the Social Sciences (SPSS) for Windows version 20.0 software. Frequency counts and percentage were generated for all variables, and statistical tests of significance were performed with correlation and regression analysis. Significance was fixed at $\mathrm{P} \leq 0.05$ and highly significant if $\mathrm{P} \leq 0.01$. Concentrations of illicit drugs in the urine samples of polysubstance users were deduced from the chromatograms using the formula;

$$
\text { Concentration of drug }(\mathrm{mg} / \mathrm{L})=\frac{\text { Concentration of Standard x Area of Drug }}{\text { Area of Standard }}
$$

\section{RESULTS}

Out of the 264 Nigerian male commercial vehicle drivers who participated in this study, $72(27.27 \%)$ were Hausas, 96 (36.36 \%) each were Igbos and Yorubas respectively. In Table 1, the data obtained from the questionnaires revealed that the lowest Mean \pm SE value for age among the three ethnicities studied was $41.47 \pm$
1.02 years (Igbo) while highest was $42.07 \pm 1.56$ years (Hausa). The highest numbers of single (33(34.4\%)) and married $(73(76.0 \%))$ drivers were obtained from Yoruba and Igbo drivers respectively. Also, the lowest numbers of single (13(18.1\%)) and married (59(81.9 $\%)$ ) drivers were obtained from Hausa drivers.

Table 1. Descriptive statistics of sociodemographic parameters in all drivers.

\begin{tabular}{lllll}
\hline \multirow{2}{*}{ Parameters } & \multirow{2}{*}{ Variables } & \multicolumn{4}{l}{ Distribution across ethnicities $(\%)$} & Yoruba (N=96) \\
\cline { 3 - 5 } & & Hausa $(\mathbf{N}=\mathbf{7 2})$ & Igbo $(\mathbf{N}=\mathbf{9 6})$ & 21.0 \\
\multirow{2}{*}{ Age (Years) } & Minimum & 19.0 & 67.0 & 72.0 \\
& Minimum & 72.0 & $41.47 \pm 1.02$ & $41.96 \pm 1.21$ \\
\hline \multirow{2}{*}{ Marital status (\%) } & Mean \pm S.E & $42.07 \pm 1.56$ & $23(24)$ & $33(34.4)$ \\
& Single & $13(18.1)$ & $73(76)$ & $63(65.6)$ \\
\hline
\end{tabular}

The study showed that $236(89.4 \%)$ were bus divers while $15(5.7 \%)$ and $13(4.9 \%)$ were car and tricycle drivers respectively. In Table 2, Igbo drivers had the highest (88 (91.7\%)) number of bus drivers while Hausas had the least $(63(87.5 \%))$. Hausa participants had the highest (28 (38.9\%) years) number of experienced divers while those with the highest number of individuals with the lowest (32 (33.3) years) years of experience were Igbo drivers. Yoruba participants had the highest (59 $(61.5 \%)$ ) number of drivers who have had accidents followed by the Hausa $(27(37.5 \%))$ and Igbo $(23(24.0 \%))$ drivers respectively.
In Table 3, Hausa drivers had the highest (54 (75.0 $\%)$ ) number of illicit drug users while the Igbo drivers had the lowest $(20(20.8 \%))$ number. Marijuana was indicated to be the most used drug by the drivers compared to other psychoactive drugs across all ethnicities; Hausa (36(50.0\%)), Igbo (13(13.5\%)) and Yoruba $(36(37.5 \%))$. Across all ethnic groups, increased energy was observed to the major reason why drivers used illicit drugs. Additionally, smoking was indicated as the major mode of administration of illicit drugs among the drivers across all ethnic groups. Feeling high after drug use was indicated as the major post administration effect of illicit drug use across all ethnic groups. 
Table 2. Descriptive statistics of driving experience and accident history parameters in all ethnicities.

\begin{tabular}{|c|c|c|c|c|}
\hline \multirow{2}{*}{ Parameters } & \multirow{2}{*}{ Variables } & \multicolumn{3}{|c|}{ Frequency across ethnicities ( \%) } \\
\hline & & Hausa & Igbo & Yoruba \\
\hline \multirow{3}{*}{ Vehicle type } & Bus & $63(87.5)$ & $88(91.7)$ & $85(88.5)$ \\
\hline & Car & $7(9.7)$ & $0(0)$ & $8(8.3)$ \\
\hline & Tricycle & $2(2.8)$ & $8(8.3)$ & $3(3.1)$ \\
\hline \multirow{3}{*}{ Driving experience } & $<5$ years & $14(19.4)$ & $32(33.3)$ & $22(22.9)$ \\
\hline & $<10$ years & $30(41.7)$ & 38 (39.6) & $52(54.2)$ \\
\hline & $\geq 10$ years & $28(38.9)$ & $26(27.1)$ & $22(22.9)$ \\
\hline \multirow{2}{*}{ Accident history } & Yes & $27(37.5)$ & $23(24)$. & $59(61.5)$ \\
\hline & No & $45(62.5)$ & $73(76)$ & $37(38.5)$ \\
\hline \multirow{4}{*}{ Number of times } & $<5$ times & $20(27.8)$ & $20(20.8)$ & $50(52.1)$ \\
\hline & $<10$ times & $5(6.9)$ & $3(3.1)$ & $8(8.3)$ \\
\hline & $\geq 10$ times & $2(2.8)$ & $0(0)$ & $1(1.0)$ \\
\hline & None & $45(62.5)$ & $73(76)$ & $37(38.5)$ \\
\hline
\end{tabular}

Table 3. Descriptive statistics of psychoactive drug use history parameters in all drivers.

\begin{tabular}{|c|c|c|c|c|}
\hline \multirow{2}{*}{ Parameter } & \multirow{2}{*}{ Variable } & \multicolumn{3}{|c|}{ Frequency across ethnicities (\%) } \\
\hline & & Hausa $(\mathrm{N}=72)$ & $\operatorname{Igbo}(\mathrm{N}=96)$ & Yoruba $(\mathrm{N}=96)$ \\
\hline \multirow{2}{*}{ Have you used drugs before? } & Yes & $54(75)$ & $20(20.8)$ & $37(38.5)$ \\
\hline & No & $18(25)$ & $76(79.2)$ & $59(61.5)$ \\
\hline \multirow{4}{*}{ Type of drug } & Maijuana & $36(50)$ & $13(13.5)$ & $36(37.5)$ \\
\hline & Tablet & $18(25)$ & $6(6.3)$ & $1(1)$ \\
\hline & Syrup & $0(0)$ & $1(1)$ & $0(0)$ \\
\hline & None & $18(25)$ & $76(79.2)$ & $59(61.5)$ \\
\hline \multirow{6}{*}{ Reason for drug use } & Alertness & $6(8.3)$ & $4(4.2)$ & $10(10.4)$ \\
\hline & Increased energy & $26(36.1)$ & $10(10.4)$ & $19(19.8)$ \\
\hline & Improve sexual performance & $2(2.8)$ & $1(1.0)$ & $4(4.2)$ \\
\hline & Pleasure & $18(25)$ & $2(2.1)$ & $1(1)$ \\
\hline & Peer pressure & $2(2.8)$ & $3(3.1)$ & $3(3.1)$ \\
\hline & None & $18(25)$ & $76(79.2)$ & $59(61.5)$ \\
\hline \multirow{3}{*}{ Did you use it in the past 90 days? } & Yes & $54(75)$ & $18(18.8)$ & $36(37.5)$ \\
\hline & No & $0(0)$ & $2(2.1)$ & $1(1)$ \\
\hline & None & $18(25)$ & $76(79.2)$ & $59(61.5)$ \\
\hline \multirow{4}{*}{ Frequency of use } & Everyday & $35(48.6)$ & $18(18.8)$ & $32(33.3)$ \\
\hline & Weekends & $8(11.1)$ & $2(2.1)$ & $4(4.2)$ \\
\hline & Weekly & $11(15.3)$ & $0(0)$ & $1(1)$ \\
\hline & None & $18(25)$ & $76(79.2)$ & $59(61.5)$ \\
\hline \multirow{5}{*}{ Last time used } & Last $24 \mathrm{~h}$ & $33(45.8)$ & $19(19.8)$ & $34(35.4)$ \\
\hline & 1-3 day(s) ago & $12(16.7)$ & $1(1)$ & $2(2.1)$ \\
\hline & Last week & $9(12.5)$ & $0(0)$ & $\mathrm{O}(0)$ \\
\hline & $\geq 1$ month & $0(0)$ & $0(0)$ & $1(1)$ \\
\hline & None & $18(25)$ & $76(79.2)$ & $59(61.5)$ \\
\hline \multirow{3}{*}{ Mode of administration } & Smoking & $35(48.6)$ & $14(14.6)$ & $36(37.5)$ \\
\hline & Ingestion & $19(26.4)$ & $6(6.3)$ & $1(1.0)$ \\
\hline & None & $18(25)$ & $76(79.2)$ & $59(61.5)$ \\
\hline \multirow{3}{*}{ Do you feel addicted to it? } & Yes & $13(18.1)$ & $6(6.3)$ & $15(15.6)$ \\
\hline & No & $41(56.9$ & $14(14.6)$ & $22(22.9)$ \\
\hline & None & $18(25)$ & $76(79.2)$ & $59(61.5)$ \\
\hline \multirow{3}{*}{ How do you get it? } & Park sellers & $52(72.2)$ & $14(14.6)$ & $29(30.2$ \\
\hline & Private suppliers & $2(2.8)$ & $6(6.3)$ & $8(8.3)$ \\
\hline & None & $18(25)$. & $76(79.2)$ & $59(61.5)$ \\
\hline \multirow{3}{*}{ Is the drug costly? } & Yes & $9(12.5)$ & $3(3.1)$ & $3(3.1)$ \\
\hline & No & $45(62.5)$ & $17(17.7)$ & 34 935.4) \\
\hline & None & $18(25)$ & $76(79.2)$ & $59(61.5)$ \\
\hline \multirow{3}{*}{ Can you do without it? } & Yes & $21(29.2)$ & $12(12.5)$ & $23(24)$ \\
\hline & No & $33(45.8)$ & $8(8.3)$ & $14(14.5)$ \\
\hline & None & $18(25)$ & $76(79.2)$ & $59(61.5)$ \\
\hline \multirow{3}{*}{ Feeling after use } & High & $35.00(48.6)$ & $19(19.8)$ & 36 937.5) \\
\hline & Sleepy & $19(26.4)$ & $1(1.0)$ & $1(1)$ \\
\hline & None & $18(25)$ & $76(79.2)$ & $59(61.5)$ \\
\hline
\end{tabular}


Table 4 shows the distribution of the participants by psychoactive drug use history. Out of the 264 participants across all ethnic groups, 111(42.0\%) had used illicit drugs in recent times while $153(58.0 \%)$ did not, 55(20.8\%) abused illicit drugs for increased energy and $85(32.2 \%)$ used illicit drugs daily. Additionally, $90(34.1 \%)$ of the respondents indicated that they felt high after taking illicit drugs.

Table 4. Distribution of the respondents by psychoactive drug use history.

\begin{tabular}{|c|c|c|}
\hline Parameter & Variable & Frequency $(\%)$ \\
\hline \multirow{2}{*}{ Have you used drugs before? } & Yes & $111(42)$ \\
\hline & No & $153(58)$ \\
\hline \multirow{6}{*}{ Reason for drug use } & Alertness & $20(7.6)$ \\
\hline & Increased energy & $55(20.8)$ \\
\hline & Improve sexual performance & $7(2.7)$ \\
\hline & Pleasure & $21(8)$ \\
\hline & Peer pressure & $8(3)$ \\
\hline & None & $153(58)$ \\
\hline \multirow{4}{*}{ Frequency of use } & Everyday & $85(32.2)$ \\
\hline & Weekends & $13(4.9)$ \\
\hline & Weekly & $13(4.9)$ \\
\hline & None & $153(58)$ \\
\hline \multirow{3}{*}{ Mode of administration } & Smoking & $85(32.2)$ \\
\hline & Ingestion & $26(9.8)$ \\
\hline & None & $153(58)$ \\
\hline \multirow{3}{*}{ Do you feel addicted to it? } & Yes & $35(13.3)$ \\
\hline & No & $76(28.8)$ \\
\hline & None & $153(58)$ \\
\hline \multirow{3}{*}{ How do you get it? } & Park sellers & $94(35.6)$ \\
\hline & Private suppliers & $17(6.4)$ \\
\hline & None & $153(58)$ \\
\hline \multirow{3}{*}{ Feeling after use } & High & $90(34.1)$ \\
\hline & Sleepy & $21(8)$ \\
\hline & None & $153(58)$ \\
\hline
\end{tabular}

$\mathrm{N}=264$

Pearson correlation analysis was carried out to determine the relationship between sociodemographics, driving experience history and psychoactive drug use history. Across all ethnic groups (Table 5), ethnicity correlated with drug use, drug name, reason for drug use, frequency of drug use, feeling of addiction, acquisition of drugs and cost and post administration effects of drug use at 0.01 level of significance. However, there was no correlation between ethnicity and mode of administration ( $\mathrm{p} \geq 0.05)$. Accident history correlated with drug use, drug name, reason for drug use, frequency of drug use, mode of administration, feeling of addiction, acquisition of drugs and cost and post administration effects of drug use at 0.01 level of significance.

All urine samples were analysed using drug test kits. In Table 6, marijuana 77(29.2\%) was the most abused substance followed by benzodiazepine 34(12.9\%) across all ethnicities studied. Marijuana had the highest $32(44.4 \%)$ number of users among the Hausa drivers, followed by benzodiazepine $28(38.9 \%$ ) while the least was morphine $3(4.2 \%)$. For the Igbo drivers, the use of marijuana was the highest $14(14.6 \%)$, followed by methadone $10(10.4 \%)$ with the least being morphine and phencyclidine 1(1\%). Amongst the Yoruba drivers, marijuana was also most abused substance 31(32.3\%) followed by benzodiazepine $4(4.2 \%)$ while the least abused drugs were barbiturate and methadone 1(1\%) each.

Across all ethnicities, Hausa drivers had the highest number of polysubstance users compared to other ethnic groups as shown in Table 7. 
Table 5. Correlation between sociodemographics, driving experience history and psychoactive drug use history in ethnic groups.

\begin{tabular}{|c|c|c|c|c|c|c|c|c|c|c|c|c|}
\hline 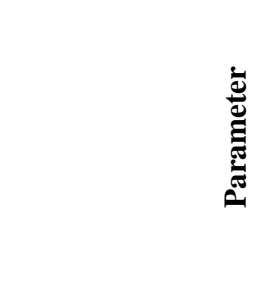 & 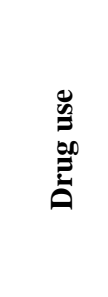 & 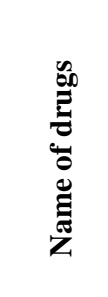 & 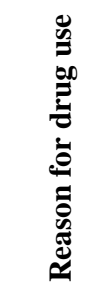 & 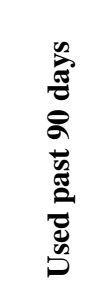 & 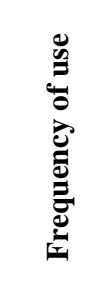 & 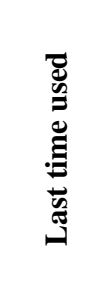 & 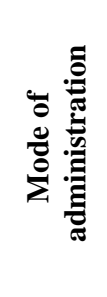 & 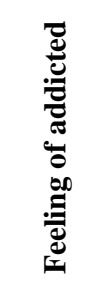 & 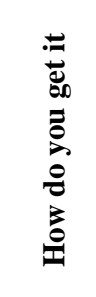 & 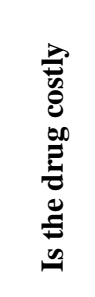 & 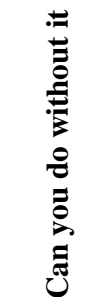 & 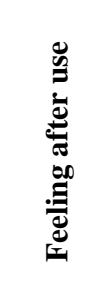 \\
\hline Age & 0.02 & -0.02 & -0.09 & -0.02 & 0.01 & -0.01 & -0.02 & -0.05 & -0.00 & 0.00 & 0.03 & -0.04 \\
\hline Ethnicity & $0.26^{* *}$ & $0.20^{* *}$ & $0.18^{* *}$ & $0.26^{* *}$ & $0.19^{* *}$ & $0.22^{* * *}$ & 0.12 & $0.18^{* *}$ & $0.39^{* *}$ & $.026^{* *}$ & $0.17^{* *}$ & $0.19^{* *}$ \\
\hline Marital Status & -0.02 & -0.02 & -0.07 & -0.04 & 0.02 & 0.00 & -0.03 & -0.07 & -0.03 & -0.02 & -0.02 & -0.03 \\
\hline Vehicle Type & -0.04 & -0.02 & .028 & 0.01 & -0.05 & -0.05 & -0.02 & -0.02 & 0.01 & -0.04 & -0.08 & -0.05 \\
\hline $\begin{array}{r}\text { Driving } \\
\text { experience }\end{array}$ & -0.01 & -0.04 & -0.09 & -0.05 & -0.02 & -0.02 & -0.03 & -0.02 & -0.03 & 0.00 & -0.00 & -0.03 \\
\hline Accident history & $0.33^{* *}$ & $0.37^{* *}$ & $0.34^{* *}$ & $0.31^{* *}$ & $0.34^{* *}$ & $0.35^{* *}$ & $0.37^{* *}$ & $0.36^{* *}$ & $0.18^{* *}$ & $0.29^{* *}$ & $0.31^{* *}$ & $0.36^{* *}$ \\
\hline Number of times & $0.26^{* *}$ & $0.30^{* *}$ & $0.28^{* *}$ & $0.25^{* *}$ & $0.26^{* *}$ & $0.27^{* *}$ & $0.30^{* *}$ & $0.30^{* *}$ & $0.14^{*}$ & $0.25^{* *}$ & $0.24^{* *}$ & $0.28^{* *}$ \\
\hline
\end{tabular}

* = Correlation is significant at the 0.05 level.

$* *=$ Correlation is significant at the 0.01 level

Table 6. Frequency distribution of drugs across ethnic groups rom toxicological testing.

\begin{tabular}{|c|c|c|c|c|c|c|c|c|c|c|}
\hline \multirow[b]{2}{*}{ Ethnicity } & \multicolumn{10}{|c|}{ Drug } \\
\hline & $\begin{array}{l}\text { THC } \\
(\%)\end{array}$ & $\begin{array}{l}\text { MOP } \\
(\%)\end{array}$ & $\begin{array}{l}\text { BAR } \\
(\%)\end{array}$ & $\begin{array}{l}\text { COC } \\
(\%)\end{array}$ & $\begin{array}{l}\text { OXY } \\
(\%)\end{array}$ & $\begin{array}{l}\text { AMP } \\
(\%)\end{array}$ & $\begin{array}{l}\text { MET } \\
(\%)\end{array}$ & $\begin{array}{l}\text { PCP } \\
(\%)\end{array}$ & $\begin{array}{l}\text { BZO } \\
(\%)\end{array}$ & $\begin{array}{l}\text { MTD } \\
(\%)\end{array}$ \\
\hline $\begin{array}{l}\text { Hausa } \\
(\mathrm{N}=72)\end{array}$ & $\begin{array}{l}32 \\
(44.4)\end{array}$ & $\begin{array}{l}3 \\
(4.2)\end{array}$ & $\begin{array}{l}0 \\
(0)\end{array}$ & $\begin{array}{l}0 \\
(0)\end{array}$ & $\begin{array}{l}0 \\
(0)\end{array}$ & $\begin{array}{l}0 \\
(0)\end{array}$ & $\begin{array}{l}0 \\
(0)\end{array}$ & $\begin{array}{l}4 \\
(5.6)\end{array}$ & $\begin{array}{l}28 \\
(38.9)\end{array}$ & $\begin{array}{l}0 \\
(0)\end{array}$ \\
\hline $\begin{array}{l}\text { Igbo } \\
(\mathrm{N}=96)\end{array}$ & $\begin{array}{l}14 \\
(14.6)\end{array}$ & $\begin{array}{l}1 \\
(1)\end{array}$ & $\begin{array}{l}0 \\
(0)\end{array}$ & $\begin{array}{l}0 \\
(0)\end{array}$ & $\begin{array}{l}0 \\
(0)\end{array}$ & $\begin{array}{l}0 \\
(0)\end{array}$ & $\begin{array}{l}0 \\
(0)\end{array}$ & $\begin{array}{l}1 \\
(1)\end{array}$ & $\begin{array}{l}2 \\
(2.10)\end{array}$ & $\begin{array}{l}10 \\
(10.4)\end{array}$ \\
\hline $\begin{array}{l}\text { Yoruba } \\
(\mathrm{N}=96)\end{array}$ & $\begin{array}{l}31 \\
(32.3)\end{array}$ & $\begin{array}{l}3 \\
(3.1)\end{array}$ & $\begin{array}{l}1 \\
(1)\end{array}$ & $\begin{array}{l}0 \\
(0)\end{array}$ & $\begin{array}{l}0 \\
(0)\end{array}$ & $\begin{array}{l}0 \\
(0)\end{array}$ & $\begin{array}{l}2 \\
(2.1)\end{array}$ & $\begin{array}{l}2 \\
(2.1)\end{array}$ & $\begin{array}{l}4 \\
(4.2)\end{array}$ & $\begin{array}{l}1 \\
(1)\end{array}$ \\
\hline $\begin{array}{l}\text { All ethnicities } \\
(\mathrm{N}=264)\end{array}$ & $\begin{array}{l}77 \\
(29.2)\end{array}$ & $\begin{array}{l}7 \\
(2.7)\end{array}$ & $\begin{array}{l}1 \\
(0.4)\end{array}$ & $\begin{array}{l}0 \\
(0)\end{array}$ & $\begin{array}{l}0 \\
(0)\end{array}$ & $\begin{array}{l}0 \\
(0)\end{array}$ & $\begin{array}{l}2 \\
(0.8)\end{array}$ & $\begin{array}{l}7 \\
(2.7)\end{array}$ & $\begin{array}{l}34 \\
(12.9)\end{array}$ & $\begin{array}{l}11 \\
(4.2)\end{array}$ \\
\hline $\begin{array}{ll}\text { Key: } & \text { THC }=\mathrm{T} \\
& \mathrm{AMP}=\end{array}$ & $\begin{array}{l}\text { hydroca } \\
\text { hetamir }\end{array}$ & & $\begin{array}{l}=\text { Barbi } \\
=\text { Meth }\end{array}$ & & $\begin{array}{l}=\text { Pher } \\
=\text { Ben }\end{array}$ & $\begin{array}{l}\text { Ine } \\
\text { epines }\end{array}$ & $\begin{array}{l}\mathrm{COC}=\mathrm{Coc} \\
\mathrm{OXY}=\mathrm{Ox}\end{array}$ & & $\begin{array}{l}\mathrm{DP}=\mathrm{Mo} \\
\mathrm{ET}=\mathrm{Met}\end{array}$ & phetamine \\
\hline
\end{tabular}

Table 7. Frequency distribution of polysubstance users across all ethnic groups.

\begin{tabular}{|c|c|c|c|c|c|c|c|}
\hline \multirow[b]{2}{*}{ Ethnicity } & \multicolumn{7}{|c|}{ Drug } \\
\hline & $\begin{array}{l}\text { BZO+THC } \\
(\%)\end{array}$ & $\begin{array}{l}\text { THC+PCP } \\
(\%)\end{array}$ & $\begin{array}{l}\text { THC+MOP } \\
(\%)\end{array}$ & $\begin{array}{l}\text { BZO+THC+PCP } \\
(\%)\end{array}$ & $\begin{array}{l}\text { BZO+THC+MOP } \\
(\%)\end{array}$ & $\begin{array}{l}\text { PCP+THC } \\
+\mathrm{MOP}(\%)\end{array}$ & $\begin{array}{l}\text { BZO+MOP+ } \\
\text { PCP }(\%)\end{array}$ \\
\hline $\begin{array}{l}\text { Hausa } \\
(\mathrm{N}=72)\end{array}$ & $\begin{array}{l}1 \\
(1.4)\end{array}$ & $\begin{array}{l}1 \\
(1.4)\end{array}$ & $\begin{array}{l}0 \\
(0)\end{array}$ & $\begin{array}{l}3 \\
(4.2)\end{array}$ & $\begin{array}{l}2 \\
(2.8)\end{array}$ & $\begin{array}{l}0 \\
(0)\end{array}$ & $\begin{array}{l}1 \\
(1.4)\end{array}$ \\
\hline $\begin{array}{l}\text { Igbo } \\
(\mathrm{N}=96)\end{array}$ & $\begin{array}{l}2 \\
(2.1)\end{array}$ & $\begin{array}{l}1 \\
(1)\end{array}$ & $\begin{array}{l}0 \\
(0)\end{array}$ & $\begin{array}{l}0 \\
(0)\end{array}$ & $\begin{array}{l}0 \\
(0)\end{array}$ & $\begin{array}{l}0 \\
(0)\end{array}$ & $\begin{array}{l}0 \\
(0)\end{array}$ \\
\hline $\begin{array}{l}\text { Yoruba } \\
(\mathrm{N}=96)\end{array}$ & $\begin{array}{l}1 \\
(1)\end{array}$ & $\begin{array}{l}0 \\
(0)\end{array}$ & $\begin{array}{l}2 \\
(2.1)\end{array}$ & $\begin{array}{l}1 \\
(1)\end{array}$ & $\begin{array}{l}1 \\
(1)\end{array}$ & $\begin{array}{l}1 \\
(1)\end{array}$ & $\begin{array}{l}0 \\
(0)\end{array}$ \\
\hline $\begin{array}{l}\text { All ethnicities } \\
N=264\end{array}$ & $\begin{array}{l}4 \\
(1.5)\end{array}$ & $\begin{array}{l}2 \\
(0.8)\end{array}$ & $\begin{array}{l}2 \\
(0.8)\end{array}$ & $\begin{array}{l}4 \\
(1.5)\end{array}$ & $\begin{array}{l}3 \\
(1.1)\end{array}$ & $\begin{array}{l}1 \\
(0.4)\end{array}$ & $\begin{array}{l}1 \\
(0.4)\end{array}$ \\
\hline
\end{tabular}

Key: $\quad$ THC $=$ Tetrahydrocabinnol, $\mathrm{BZO}=$ Benzodiazepine, $\mathrm{MOP}=$ Morphine, $\mathrm{PCP}=$ Phencyclidine

Chromatographic analysis was performed using the urine samples of participants who tested positive to two or more drugs. Table 8 shows that the polysubstance users used mainly marijuana, benzodiazepine, morphine and phencyclidine. Across all ethnicities, the Hausa drivers had the highest concentration of psychoactive substances in their urine samples. The chromatographs did not show THC only in samples H14, H17 and IG58. Tetrahydrocabinnol and BZO only were not detected by HPLC in samples H53 and H66. In samples H16 and Y22, BZO only and MOP only were not detected by HPLC respectively. Also, BZO and MOP were not detected by HPLC in sample H07 but PCP was detected. 
Table 8. Concentrations of different drugs in polysubstance users.

\begin{tabular}{|c|c|c|c|c|c|}
\hline \multirow{2}{*}{ Ethnicity } & \multirow{2}{*}{ Subject } & \multicolumn{4}{|c|}{ Drug concentrations in urine $(\mathrm{mg} / \mathrm{L})$} \\
\hline & & PCP & THC & BZO & MOP \\
\hline \multirow{8}{*}{ Hausa } & H07 & 1321.3 & 671.7 & 0 & 0 \\
\hline & $\mathrm{H} 12$ & 0 & 210.6 & 15.9 & 0 \\
\hline & $\mathrm{H} 14$ & 0 & 0 & 0.02 & 0 \\
\hline & H16 & 0 & 0.2 & 0 & 0.5 \\
\hline & $\mathrm{H} 17$ & 0 & 0 & 0.1 & 93 \\
\hline & $\mathrm{H} 40$ & 10.7 & 8 & 0. & 0 \\
\hline & H53 & 122.9 & 0 & 0 & 0 \\
\hline & H66 & 167.4 & 0 & 0 & 0 \\
\hline \multirow{3}{*}{ Igbo } & IG25 & 34.7 & 8.7 & 0 & 0 \\
\hline & IG36 & 0 & 0.9 & 1.2 & 0 \\
\hline & IG58 & 0 & 0 & 2.3 & 0 \\
\hline \multirow{6}{*}{ Yoruba } & Y03 & 108.1 & 25.6 & 0 & 1.2 \\
\hline & Y04 & 0 & 19.5 & 0 & 32.8 \\
\hline & Y06 & 0.02 & 0.8 & 1.2 & 0 \\
\hline & Y10 & 0 & 0.8 & 1.5 & 0 \\
\hline & Y21 & 0 & 0.1 & 0.3 & 0.2 \\
\hline & $\mathrm{Y} 22$ & 0 & 0.1 & 0 & 0 \\
\hline Standards: & \multicolumn{2}{|c|}{$\begin{array}{l}\mathrm{PCP}=(2.5) \\
\mathrm{MOP}=(3.99)\end{array}$} & \multicolumn{2}{|c|}{$\begin{array}{l}\mathrm{THC}=(2.4) \\
\mathrm{BZO}=(2.39)\end{array}$} & \\
\hline Key: & \multicolumn{2}{|c|}{$\begin{array}{l}\text { MOP }=\text { Morphine } \\
\text { BZO = Benzodiazepines } \\
\mathrm{H}=\text { Hausa } \\
\mathrm{Y}=\text { Yoruba }\end{array}$} & \multicolumn{3}{|c|}{$\begin{array}{l}\text { PCP = Phencyclidine } \\
\text { THC = Tetrahydrocabinnol } \\
\text { IG = Igbo }\end{array}$} \\
\hline
\end{tabular}

\section{DISCUSSION}

The ages of the commercial vehicle divers who participated in this study ranged from 19 to 72 years old. Majority of the drivers studied fell with the age range (15-64 years) given by UNODC, (2018) as the age range that has the highest prevalence of drug use. Across all ethnic groups, $111(42.0 \%)$ of the respondents had used illicit drugs in recent times and this value is higher than what was reported in the report of Iroanya et al., (2018) who reported that only $5.7 \%$ of their subjects had taken other stimulants aside alcohol in recent times. The study revealed that $55(20.8 \%)$ abused illicit drugs for increased energy, 20(7.6\%) for alertness, 21(8.0\%) for pleasure and $85(32.2 \%)$ used illicit drugs daily. Additionally, $90(34.1 \%)$ and $21(8.0 \%)$ of the respondents indicated that they felt high or sleepy respectively after taking illicit drugs. These findings agree with the report of Yunusa et al., (2017) who stated that the desires to relax/sleep after a hard days job (84.8 $\%)$, work hard $(48 \%)$, relieve stress $(81 \%)$, relieve anxiety $(66.5 \%)$ and pleasure $(72 \%)$ were the major factors associated with the abuse of substances by the respondent.

This study revealed that $52(72.22 \%)$ of drivers from the Hausa ethnicity used one or more psychoactive substance(s) and this agrees with the study of Yunusa et al., (2017) who reported that eight out of every ten (81.1 $\%)$ of drivers who participated in their study has abused a substance. This study showed that 25(26.04 \%) of Igbo drivers used one or more $\operatorname{drug}(\mathrm{s})$ and this is lower than the findings of Aniedu and Okonkwo, (2008) who reported the prevalence of psychoactive drug use amongst taxi drivers to be $85.4 \%$. It was also observed that $36(37.5 \%)$ of the Yoruba drivers also used one or more psychotropic substance(s) and this is also higher than the report of Iroanya et al, (2018) who reported that $24.6 \%$ of their participants tested positive to at least one psychoactive drug in a study conducted at two bus stations in Lagos state, Nigeria. Additionally, 113(42.8) of the participants across all ethnic groups tested positive to one or more psychoactive substance(s). This is lower than the finding of Hamman et al., (2018) who documented that $57.7 \%$ of the subjects tested positive for substance abuse in a study conducted among professional minibus drivers at Zagazig city, Sharqia Governorate, Egypt. The study showed that the substances commonly used by the drivers were marijuana (29.2 \%), benzodiazepine (12.9 \%), methadone $(4.2 \%)$, phencyclidine $(2.7 \%)$, morphine $(2.7 \%)$ and Methamphetamine $(0.8 \%)$. This is significantly higher than the findings of Bogstrand et al., (2012) who reported that most commonly found substances among drivers in normal traffic were Cannabis $(0.7 \%)$, benzodiazepines $(0.8 \%)$, methadone $(0.1 \%)$, methamphetamine $(0.2 \%)$ and Morphine $(0.0$ $\%)$. From this study, marijuana was the most used drugs among the drivers who participated in the study. This agrees with the report of UNODC, (2018) which reported that Cannabis was the most commonly used drug in the Nigerian population. Hamman et al., (2018) also reported that the most common abused substance was cannabis which represented $80 \%$ of the positive participants. Cannabis is the world's most commonly used illicit drug which occupies fourth place in worldwide popularity amongst psychoactive drugs, after caffeine, nicotine and alcohol (Vij, 2011). It was also observed from this study that the use of benzodiazepine by the subjects was also common after marijuana's use. This disagrees with the report UNODC (2018) which stated that cannabis' use was followed by opioids. Interestingly, the use of methodone was highest among the Igbo drivers. This disagrees with the findings of Aniedu and Okonkwo, (2008) who reported that the commonest drugs used by taxi drivers in Enugu were alcohol, tobacco and central nervous system stimulants such as coffee and kolanut. It was observed that none of the drivers across all ethnic groups tested positive to cocaine, oxycodone and amphetamine. This might be due to the unavailability of these drugs within their environments, moreover, they are also very expensive. This however is in disagreement with the findings of Dini et al., (2019) who documented that $21.3 \%$ and 2.2 $\%$ of the truck drivers who took part in their study consumed amphetamine and cocaine respectively. This study revealed that the Hausa drivers had the highest number of polysubstance users. The concentrations of PCP, THC and BZO were generally higher in the Hausa 
drivers than other ethnic groups. This agrees with the report of NDLEA (2011) regarding the urgent need for coordination and collaboration between tiers of government to tackle the scourge of drug abuse in the Northern Nigeria. It has been predicted that the use of illicit drugs kill around 0.2 million people each year, shattering families and bringing misery to thousands of other people. Illicit drugs undermine economic and social development and contribute to crime, instability, insecurity and the spread of HIV (NDLEA, 2011).

\section{CONCLUSION AND RECOMMENDATION}

The prevalence of hypertension among the Nigerian male commercial drivers in the three major ethnicities studied is high and of public health importance. The threat of drug abuse is real and enormous. Hence, the Nigerian government and relevant non-governmental organizations should come up with viable strategies on how to identify areas with high drug addiction prevalence, train specialist staff to handle drug abuse issues, educate drivers and the public on the negative effects of drug abuse. This study has revealed that a sizable proportion of the Nigerian male commercial drivers operating in the selected bus stations in Anambra, Abia, Kaduna, Sokoto, Oyo and Lagos States, Nigeria use psychoactive substances as diagnosed by the drug kits. It is very crucial for the Nigerian anti-drug agency (National Drug Law Enforcement Agency (NDLEA)) to develop new tactics in conducting a repressive drug war, and make attempts to understand the psychological world of addicts in order to formulate appropriate policies against drug abuse. There can be no better time and appropriate turning point than now.

Conflict of interest: The author declares that there are no conflicts of interest concerning the publication of this article.

\section{REFERENCES}

Akanni, O. O. and Adayonfo, E. O. 2015. Correlates of psychoactive substance use among Nigerian Adolescents. Sahel Medical Journal. 18(4): 192 - 199.

Adekoya, B. J., Adekoya, A. O., Adepoju, F. G. and Owoeye. J. F. A. 2011. Driving under influence among long distance commercial drivers in Ilorin, Nigeria. International Journal of Biological and Medical Research. 2(4): 870 - 873.

Aniebu, P. N. and Okonkwo, K. O. B. 2008. Prevalence of psychoactive drug use by Taxi drivers in Nigeria. Journal of College of Medicine. 13: 48-52

Bogstrand, S. T., Normann, P. T., Rossow, I, Larsen, M., Morland, J. and Ekeberg, O. 2011. Prevalence of alcohol and other substances of abuse among injured patients in a Norwegian emergency department. Drug and Alcohol Dependence. 117:132-138.

Bogstrand, S. T., Gjerde, H., Normann, P. T., Rossow, and Ekeberg, O. 2012. Alcohol, psychoactive substances and non- fatalroad traffic accidents - a case-control study. BMC Public Health. 12:734.

Dini, G., Bragazzi, N. L., Montecucco, A., Rahmani, A. and Durando, P. 2019. Psychoactive drug consumption among truck-drivers: a systematic review of the literature with metaanalysis and meta-regression. Journal of Preventive Medicine and Hygiene. 60: e124-e139.

Drummer, O. H., Kourtis, I., Beyer, J., Tayler, P., Boorman, M. and Gerostamoulos, D. 2012. The prevalence of drugs in injured drivers. Forensic Science International. 215:14-17.

European Monitoring Centre for Drugs and Drug Addiction (EMCDDA). 2012. Driving under the influence of drugs, alcohol and medicines in Europe-findings from the DRUID project. Luxembourg: Publications Office of the European Union, 58pp.

European Monitoring Centre for Drugs and Drug Addiction (EMCDDA). 2015. New psychoactive substances in Europe: innovative legal responses. Luxembourg: Publications Office of the European Union, 14pp.

Gopalakrishnan, S. 2012. A Public Health Perspective of Road Traffic Accidents. Journal of Family Medicine and Primary Care. 1(2): 144-150.

Hammam, R. A. M.., Zalat, M. M., Abdelsalam, N. M. and Mesallam, D. I. A. 2018. Substance abuse and driving behavior among professional minibus drivers at Zagazig city, Sharqia Governorate, Egypt. Egyptian Journal of Occupational Medicine. 42 (3):365-382.

Igwe, W. C, Ojinaka, N., Ejiofor, S. O., Emechebe, G. O. and Ibe, B. C. 2009. Sociodemographic correlates of psychoactive substance abuse among secondary school students in Enugu, Nigeria. European Journal of Social Sciences. 12:277-283.

Iroanya, O. O., Uguru-Okorie, C. G., Egwuatu, T. F.and Bello, A. J. 2018. Prevalence of substance abuse amongst commercial vehicle drivers in Bariga and Gbagada motor parks of Lagos State, Nigeria. Nigerian Journal of Pharmaceutical and Applied Science Research. 7(3):17-24.

Jenkins, A. J. and Caplan, Y. H. 2010. Drug testing in alternate biological specimens. Humana Press. Totowa, NJ, USA. 186 pp.

Nantulya, V. M. and Reich, M. R. 2002. The neglected epidemic: Road traffic injuries in developing countries. British Medical Journal. 324:1139-1141.

National Drug law Enforcement Agency (NDLEA). 2011. Report on incidence of drug abuse in Nigeria.

Nutt, D., King, L. A., Saulsbury, W. and Blakemore, C. 2010. Development of a rational scale to assess the harm of drugs of potential misuse. The Lancet: 1047-1053.

Olashore, A. A,. Ogunwobi, O., Totego, E. and Opondo P. 2018. Psychoactive substance use among first year students in a Botswana University: Pattern and Demographic correlates. BMC Psychiatry. 18(270): 1 - 9.

Oshodi, O. Y., Aina, O. F. and Onajole, A. T. 2010. Substance use among secondary school students in an urban setting in Nigeria: Prevalence and associated factors. African Journal of Psychiatry. 13:52-7

Pela, O. A. 1989. Patterns of adolescent psychoactive substance use and abuse in Benin City, Nigeria. Adolescent. 24(95): $569-574$

Smith, M. P. and Bluth, M. H. 2016. Forensic toxicology: an introduction. Clinics in Laboratory Medicine. 36: 753-759.

United Nations Office on Drugs and Crime. 2018. Drug Use in Nigeria. Vienna. 61pp. 
Vij, K. 2011. Forensic medicine and toxicology: principles and practice. $5^{\text {th }}$ Edition. Elsevier: $A$ division of Reed Elsevier India Private Limited. India. 445pp.

World Health Organization (WHO). 2015. Global status report on road safety. Geneva. 340pp.

Wyman, J. 2012. Principles and procedures in forensic toxicology. Clinics in Laboratory Medicine. 32:493-507.

Yunusa, U., Bello, U. L., Idris, M., Haddad, M. M. and Adamu, D. Determinants of Substance Abuse among Commercial Bus
Drivers in Kano Metropolis, Kano State, Nigeria. 2017. American Journal of Nursing Science. 6(2): 125-130.

Zarrouq, B.,El Asri, B. A., Achour, S., Rammouz, I., Aalouane, R., Lyoussi, B., Khelafa, S., Bout, A., Berhili, N., Hlal, H., Najdi, A., Nejjari, C. and El Rhazi, K. 2016. Psychoactive substances use and associated factors among middle and high school students in the North Center of Morocco: a crosssectional questionnaire survey. BMC Public Health. 16(468): $1-9$. 
THIS PAGE INTENTIONALLY LEFT BLANK 